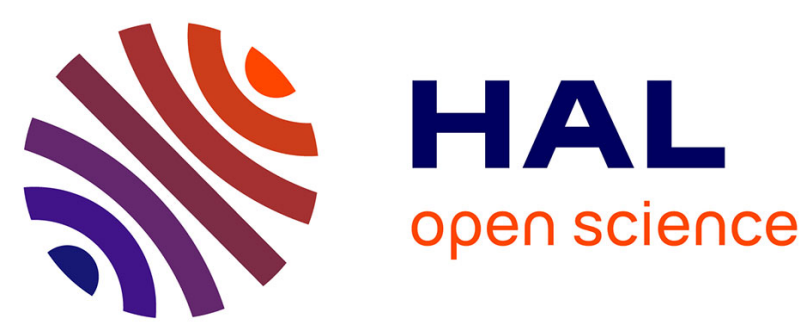

\title{
Pattern-Reconfigurable Metasurface-Antenna Array for Functional Brain Imaging Applications
}

Mohammad Ojaroudi, Stéphane Bila

\section{To cite this version:}

Mohammad Ojaroudi, Stéphane Bila. Pattern-Reconfigurable Metasurface-Antenna Array for Functional Brain Imaging Applications. 2021 15th European Conference on Antennas and Propagation (EuCAP), Mar 2021, Dusseldorf, Germany. pp.1-5, 10.23919/EuCAP51087.2021.9411500 . hal03455443

\section{HAL Id: hal-03455443 \\ https://hal-unilim.archives-ouvertes.fr/hal-03455443}

Submitted on 29 Nov 2021

HAL is a multi-disciplinary open access archive for the deposit and dissemination of scientific research documents, whether they are published or not. The documents may come from teaching and research institutions in France or abroad, or from public or private research centers.
L'archive ouverte pluridisciplinaire HAL, est destinée au dépôt et à la diffusion de documents scientifiques de niveau recherche, publiés ou non, émanant des établissements d'enseignement et de recherche français ou étrangers, des laboratoires publics ou privés. 


\title{
Pattern-Reconfigurable Metasurface-Antenna Array for Functional Brain Imaging Applications
}

\author{
Mohammad Ojaroudi ${ }^{1,2}$, Stéphane Bila ${ }^{1}$ \\ ${ }^{1}$ XLIM, UMR no7252, University of Limoges/CNRS, 123 Av. Albert Thomas, 87060 Limoges, France \\ ${ }^{2}$ Inria Lille Nord Europe, 40 Av. Halley, 59650 Villeneuve d'ascq, France \\ e-mail: mohammad.ojaroudi-parchin@inria.fr
}

\begin{abstract}
In this paper, a compact bow-tie dipole antenna with the pattern reconfigurability characteristics is presented for cerebrovascular monitoring. In order to create a pattern reconfigurable characteristic a balun-matched bow-tie antenna is equipped with a pentagonal shaped metasurface structure with five triangular meta-atoms. The proposed structure with a multi-layer phantom of the human head inside a designed matching medium are simulated in CST medium. To enhance the frequency range to have return loss less than 10 in $\mathbf{0 . 5 - 5}$ $\mathrm{GHz}$, a matching medium is used. In addition, by using these metasurface structure the realized gain is increased from $\mathbf{1 . 2 9}$ dBi to $2.38 \mathrm{dBi}$ at $3 \mathrm{GHz}$. The inserted pin-diodes on the triangular corners can provide the desired pattern reconfigurability. Hence the main beam direction can be controlled. Simulated reflection and radiation results demonstrate that the proposed meta-antenna and its pattern reconfigurability property effectively applicable for functional brain imaging applications.
\end{abstract}

Index Terms-microwave imaging system, cerebrovascular monitoring, pattern reconfigurability, pentagonal shaped metasurface structure, bow-tie dipole antenna.

\section{INTRODUCTION}

Currently brain imaging is performed with a variety of diagnostic and monitoring techniques such as computed tomography (CT), ultrasound (US), positron emission tomography (PET) scan, statistical and functional magnetic resonance imaging (fMRI), electroencephalogram (EEG), and Magnetoencephalography (MEG) [1-3]. However, CT scanners result in high accuracy, they cause ionization of biological tissue due to their radioactive emission [2]. The spatial and temporal resolution of the ultrasound techniques is not suitable for brain activities monitoring. Regarding to continuous monitoring requirements, MRI is not practical because of its mechanical complexity and bulky size [4]. In addition, patients with prostheses or pace maker are excluded due to interference between the emitted wave and the metallic components in the body [5]. In addition, fMRI scanners are excessively large and expensive, also they are not suitable for using during neurosurgery operations [6].

Over the last two decades, non-ionizing, non-invasive microwave imaging systems (MIS) have been investigated for medical diagnostics applications [7-8]. A limited number of these works have reached the stage of small-scale clinical testing, but there are still many challenges to consider as a well-established method [9-10]. In recent published papers in microwave imaging system and cognitive radar areas, we have introduced a cognitive approach based on a set of multi- shape exciting pulse-trains and beamforming techniques which have proved to be simple and efficient [11-12]. The envisioned functional microwave imaging system (FMIS) with cognitive scanning characteristic provides unique capabilities suitable for high-resolution non-invasive imaging systems [11]. In the cerebrovascular monitoring framework using ultra-wideband (UWB) radars, it is assumed that if we could detect the blood vessels dilation from received signals, it is possible to demonstrate the brain functionalities by using image reconstruction methods [12]. This is the main motivation for the radar-based perspective towards developing disruptive new methods to monitor brain activities using specifically coded ultra-wideband (UWB) signals. The development and widespread application of cognitive scanning for functional microwave imaging based ultra-wideband transceivers indeed provides an opportunity for utilizing their unique features for studying neural activities. In addition, recent developments in pseudo-noise multi-input multi-output (MIMO) radar design and advances in pattern-reconfigurable antenna array manufacturing provide the capability to package them into an autonomous portable system [13-14].

In this paper, we explore the advantages of using metasurface structure to create pattern reconfigurability of bow-tie dipole antenna for cerebrovascular monitoring applications. In this context, a novel design of compact matched bowtie antenna with a matching balun in the feedline and a pentagonal metasurface structure with five triangular-rings meta-atoms in the front of the square radiating patch is presented. Also, in order to create the desired scenario under test we used a multi-layer full head phantom and we designed a matching medium with appropriate electrical characteristics. By using these modified structures, the usable frequency bandwidth of the proposed slot antenna is progressed from $0.5 \mathrm{GHz}$ to $5 \mathrm{GHz}$. The designed square dipole antenna as principal radiator has a small size of $20 \times 20 \times 0.8 \mathrm{~mm}^{3}$, and by using the proposed metasurface structure the realized gain is increased from $1.29 \mathrm{dBi}$ to $2.38 \mathrm{dBi}$ at $3 \mathrm{GHz}$. The inserted pin-diodes on the triangular corners can provide the desired pattern reconfigurability, hence the main beam direction can be controlled. Simulated reflection coefficients and radiation patterns results demonstrate that the proposed meta-antenna and its pattern reconfigurability property effectively applicable for functional brain imaging applications. 


\section{FUNCTIONAL BRAIN IMAGING PARADIGM BASED ON ADAPTIVE MIMO BEAMFORMING}

In this paper, we present a pattern reconfigurable antenna which is applicable in envisioned cognitive scanning (CS) approach for cerebrovascular monitoring [11]. In multi-static imaging, in each stage, only one antenna is excited as a transmitter with a fixed signal, so that in the next steps, this fixed signal is used for the rest of the antennas. But in cognitive imaging, at each stage, several antennas with orthogonal signals are excited in an array form. Also, due to the change in the position of the targets in the next stages of the scan, the direction of main lobe in radiation pattern is changed towards the new position of the targets. The main argument for the superiority of $\mathrm{CS}$ for brain functional imaging is that it is more flexible for unknown pattern of brain activities than multi-static imaging and thus can be proposed more widely as a monitoring option with less contraindication. The phenomena behind this idea is that for monitoring and functional imaging we do not need to scan the entire volume of the brain and only save/exploit the data that carries pertinent information about pre-defined set of the brain activities. By identifying an activated region as a target of interest, the microwave-imaging scenario is switched to a microwave-sensing scenario. Fig. 1 shows the cerebrovascular monitoring as a functional microwave brain imaging scenario in two different multi-static imaging, and cognitive MIMO imaging frameworks.
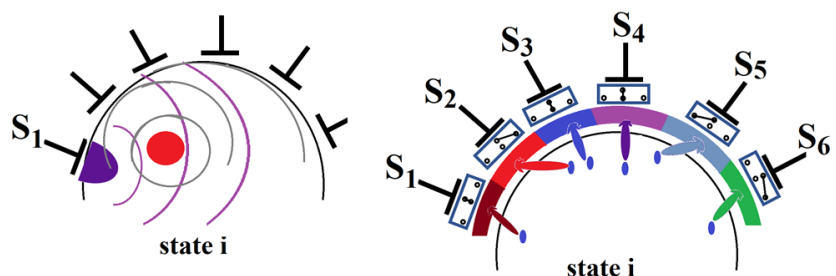

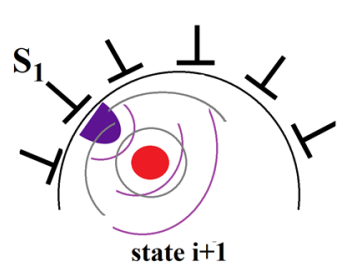

(a)

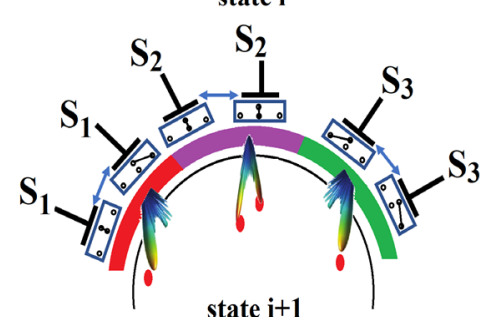

(b)
Fig. 1. Cerebrovascular monitoring as a functional microwave brain imaging scenario, (a) multi-static imaging, and (b) cognitive MIMO imaging.

Since in cerebrovascular monitoring the number of moving targets is unknown and changing over the time, the proposed adaptive MIMO framework has the waveform weighting capability to determine the adaptive beamforming of transmitter antennas. Identification and extraction of the number of multiple moving targets is the first step in creating information-based sensing framework, which is, converting - in real time - the geometrical information of the activated regions and their respective intensities to the number of targets of interest [15]. To this end, one of the main challenges confronting the proposed scenario is beam steering using metasurface subarrays and beamforming using shared aperture based on orthogonal signalling for focused imaging in elaborated sub-arrays, all capable of gathering high-resolution and high-precision backscattered signals from multiple variant targets [14]. As shown in Fig. 2, this structure includes two types of beamforming approaches: one in metasurface structures and another in beamforming by MIMO waveform diversity. Due to the special importance in controlling the radiation pattern, the antenna control mechanism has a special sensitivity in this application. In recent years, the dramatic use of metasurface has given rise to high hopes for the formation and orientation of the radiation beam in the near field [14]. On the other hand, Msequence noise radars have shown their potential in detecting small movements during several experiments [13].

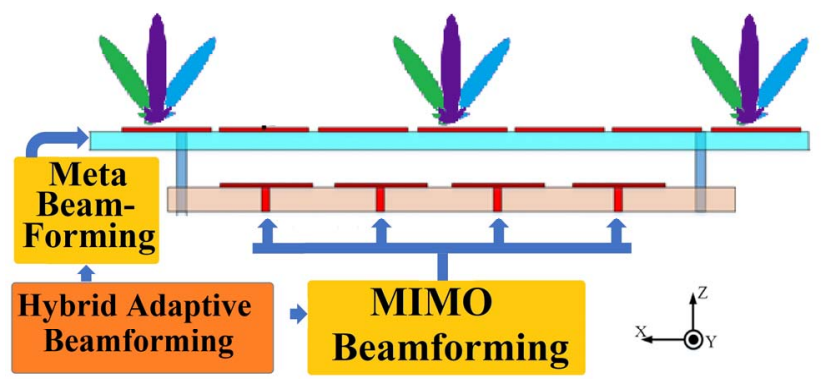

Fig. 2. Schematic illustration of the envisioned cognitive scanning paradigm based on addaptive MIMO beamfromingusing and pattern reconfigurable metasurface antennas.

\section{PATtern-ReCONFigurable Metasurface ANTENNA DESIGN AND CONFIGURATION}

In this section, in order to evaluate the required pattern reconfigurability performance for envisioned functional brain imaging system, a bow-tie dipole antenna with a pentagonal shaped metasurface including five triangular meta-atoms, is simulated in CST simulator [16] and the results are analyzed and discussed. The proposed "scenario under test" setup of the head imaging system including UWB bowtie antennas, and a multi-layer brain phantom covering by coupling medium is shown in Fig. 3. The utilized head phantom contains all anatomical details of the human head including head layers, from skin layer to white matter layer of the brain for ease of modeling and imaging. The external radius of the elliptical head phantom is $12 \mathrm{~cm}$. All electrical characteristics of the utilized head phantom's materials are given in Table I. In addition, as shown in Fig. 3, two different metasurface configurations (pentagonal, and hexagonal) are shown in the front of the bow-tie antennas. The combination of these structures has good capability to cover spherical surface of the human head. In this study we focused on the pentagonal shaped metasurface structure.

The proposed meta-antenna structure is depicted in Fig 4 which is designed based on the bow-tie antenna presented in Ref. [7] with a new design of five trangular-ring metasurface structures. The bow-tie antenna fed by a $50 \Omega$ microstrip line, which is printed on an Rogress 5880 substrate with the dimension of $22 \times 22 \mathrm{~mm}^{2}$, the dielectric constant of 2.2 , and 
the loss tangent of 0.001 . The basic antenna structure consists of a squre radiating patch, a balun type feed line. In additional the pentagonal-shaped metasurface structure with five triangular unitel-cells are shown in this Figure. Each unit cell includes a pin-diode in oredear to create pattern reconfigurability proprety. All dimensions of the antenna are given in Fig. 4.
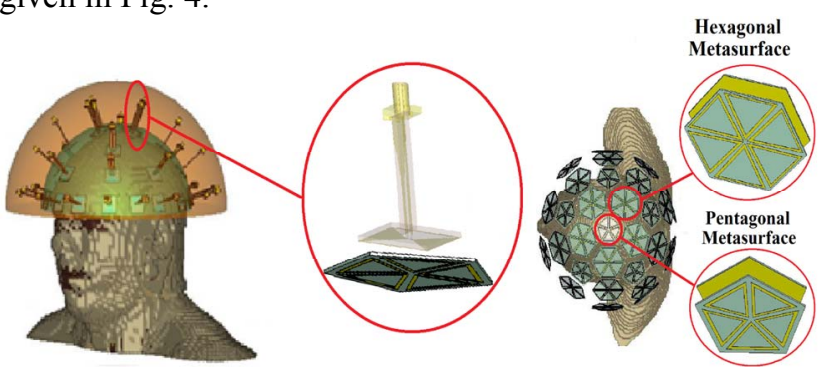

(a)

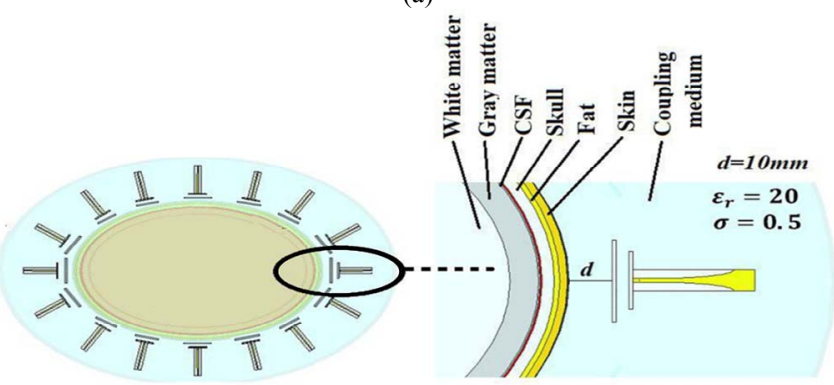

(b)

Fig. 3. (a) Meat-antenna configuration in envisioned functionl brain imaging, and (b) Simulated multi layer structure of the designed head phantom inculidning meta-antenna.

TABLE I. ELECTRICAL CHARACTERITICS OF THE MULTI-LAYER BRAIN PHANTOM

\begin{tabular}{|l|c|c|c|}
\hline \multicolumn{1}{|c|}{ Tissue } & $\begin{array}{c}\text { Thickness } \\
(\mathbf{m m})\end{array}$ & $\begin{array}{c}\text { Relative } \\
\text { Permittivity }\end{array}$ & $\begin{array}{c}\text { Conductivity } \\
(\mathbf{S} / \mathbf{m})\end{array}$ \\
\hline Skin (dry) & 2 & 40.93 & 0.89 \\
\hline Fat & 1.4 & 5.44 & 0.05 \\
\hline Skull & 4.1 & 12.36 & 0.15 \\
\hline $\begin{array}{l}\text { Cerebrospinal } \\
\text { fluid (CSF) }\end{array}$ & 0.5 & 68.43 & 2.45 \\
\hline Gray Matter & 7 & 52.28 & 0.98 \\
\hline White Matter & Inner Part & 38.57 & 0.62 \\
\hline
\end{tabular}

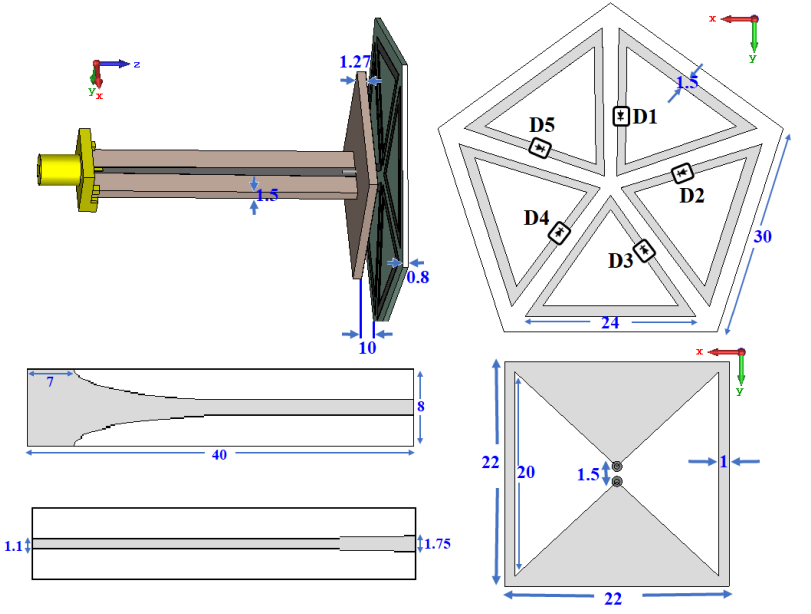

Fig. 4. The proposed meta-antenna schematic and dimensions (unit: $\mathrm{mm}$ ).

\section{RESUlTS AND DiscUSSIONS}

In this section, in order to evaluate the pattaren reconfigurability performance of the proposed bow-tie dipole antenna with a pentagonal shaped metasurface including five triangular meta-atoms, a multi-layer brain phantom is designed in SCT simulator and the results are analyzed and discussed. The first step in order to achieve required bandwidth because of presence of head phantom is designing a matching medium. By shielding antennas in the matching medium, it is possible to decrease the mismatch effects between antenna and head phantom [7]. To ensure electrical matching between antennas and internal of the region under test, a coupling medium is designed based on parametric sweep analysis of its electrical characteristics. The calculated electrical characteristics of the coupling medium are $\varepsilon_{r}=20$ and $\sigma=0.5 \mathrm{~S} / \mathrm{m}$. Fig. 5 shows the simulated return loss results for the different simulation scenarios in order to design the proposed antenna including matching medium and metasurface structure. It can be seen from Fig. 5 that by determining a good choice for permittivity and conductivity of the matching medium, the proposed meta-antenna radiates from 0.5 to $5 \mathrm{GHz}$.

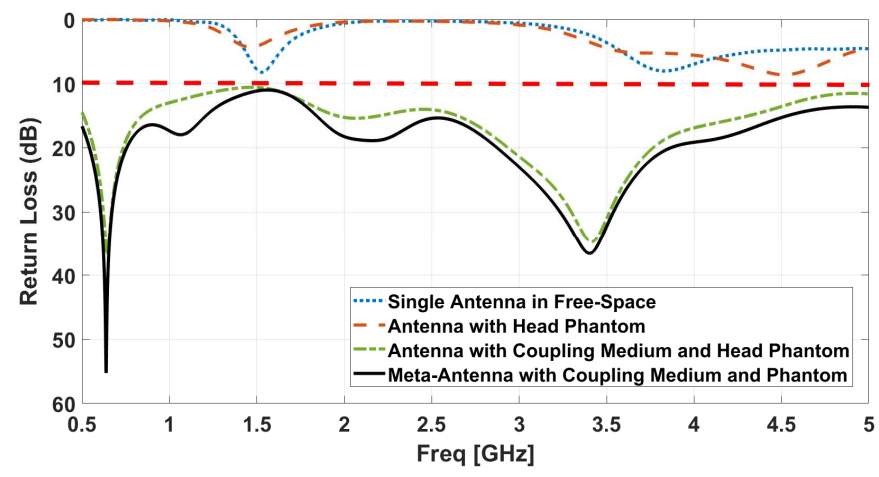

Fig. 5. Siimulated return loss results for the different simulation scenarios in order to design the proposed antenna including matching medium and metasurface structure.

The simulated surface current distribution in metasurface structure are shown in Fig. 6. As can be seen from Fig. 6, the surface current distribution is concentrated at the central vertex and their sides in each triangle-ring, indicating that the antenna radiation is very sensitive to these points. Therefore, by placing diodes in these sides, we can have more control over the radiation of the proposed antenna.

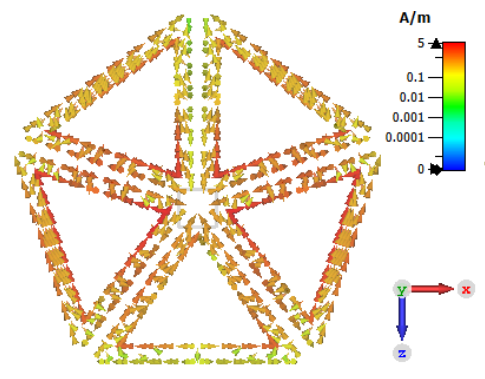

(a)

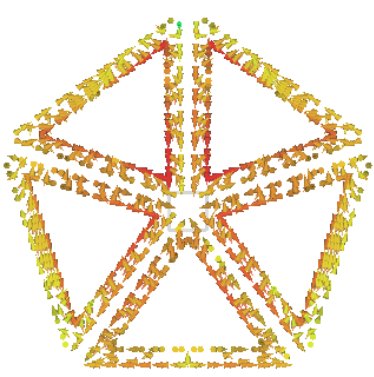

(b)
Fig. 6. Simulated surface current distribution at, (a) $1 \mathrm{GHz}$, and (b) $3 \mathrm{GHz}$. 
In order to show the effectiveness of using metasurface structure in radiation characteristics of the proposed metaantenna, the E-field radiated from the antenna in each case for two frequencies are displayed in Fig. 7. It can be seen that the using of metassurface in the proposed antenna has improved the radiative property, which has increased the penetration of the wave into the brain tissue. Also, due to the increasing in antenna gain, the field distribution is concentrated in the antenna propagation path.
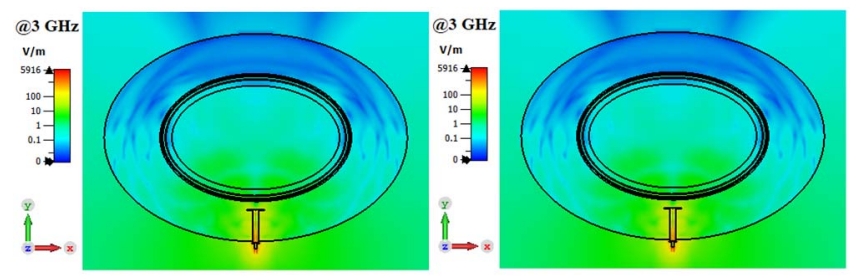

(a)

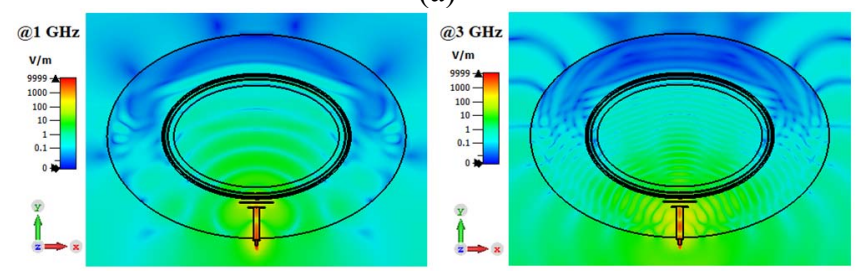

(b)

Fig. 7. Comparison of E-field pattern at 1 and $3 \mathrm{GHz}$, (a) whitout metasurface structure, and (b) with metasurface structure.

In addition, Fig. 8 illustrates the simulated radiation patterns at $3 \mathrm{GHz}$ in two different modes of with and without metasurface structure. The main purpose of presenting the radiation patterns is to demonstrate that using the metasurface structure actually improve the gain from $1.29 \mathrm{dBi}$ to $2.38 \mathrm{dBi}$. It can be seen that the radiation patterns in this situation is more directive which is an advantage for microwave imaging applications.
Farfield Realized Gain Abs (Phi=90)

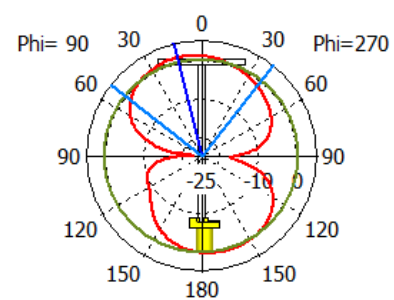

Theta / Degree vs. dBi

Frequency $=3 \mathrm{GHz}$

Main lobe magnitude $=1.29 \mathrm{dBi}$

Main lobe direction $=14.0 \mathrm{deg}$.

Angular width $(3 \mathrm{~dB})=89.8 \mathrm{deg}$.

Side lobe level $=-1.0 \mathrm{~dB}$

(a)
Farfield Realized Gain Abs (Phi=90)

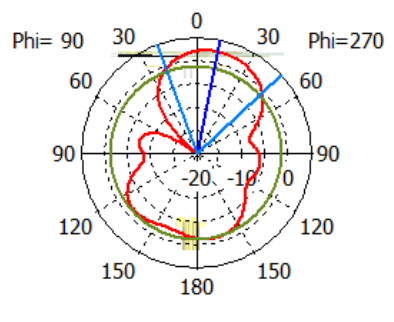

Theta / Degree vs. dBi

Frequency $=3 \mathrm{GHz}$ Main lobe magnitude $=2.38 \mathrm{~dB}$ Main lobe direction $=12.0 \mathrm{deg}$. Angular width $(3 \mathrm{~dB})=67.1 \mathrm{deg}$. Side lobe level $=-3.6 \mathrm{~dB}$

(b)
Fig. 8. Comparison of radiation pattern and realized gain at $3 \mathrm{GHz}$ (a) whitout metasurface structure, and (b) with metasurface structure.
To show the pattern reconfigurability characteristics of the proposed antenna, we have turned the diodes on and off in different modes. Four examples are optionally shown in Fig. 9. As it is clear from Fig. 9, by changing the state of the diodes, the main lobe direction of the radiation pattern can be controlled.

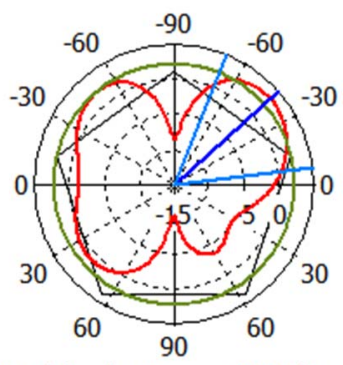

Main lobe direction $=-42.0$ deg.

(a)

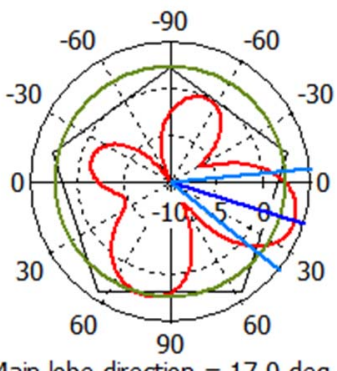

(c)

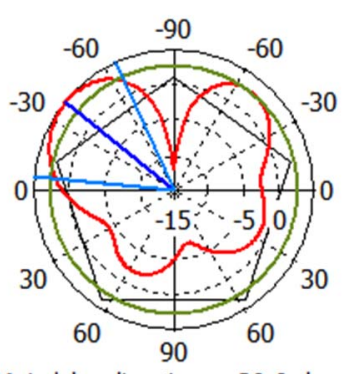

Main lobe direction $=-39.0 \mathrm{deg}$.

(b)

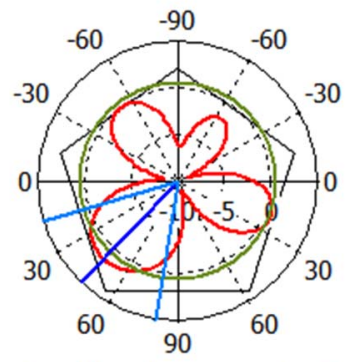

Main lobe direction $=46.0 \mathrm{deg}$.

(d)
Fig. 9. Four sample of radiation patterns regording to different states of the diodes. (a) diode 1 is off and the rest are on, (b) diode 2 is off and the rest are on (c) diode 2 and 3 are off and the rest is on, and (d) diodes 5 , 1 are on and the rest are off.

\section{CONCLUSION}

In this paper, a new design of meta-antenna with reconfigurable pattern capability for functional brain imaging applications is presented. The basic antenna for this work is a square bow-tie antenna, which by adding a metasurface structure with five triangular rings that have diodes on the sides of the triangles, has made it possible to rotate the radiation pattern. By designing a multi-layer model of human brain tissue with a suitable coupling environment, the proposed antenna radiation bandwidth is determined between 0.5-5 GHz. Simulation results such as return losses, surface current distribution, antenna gain and radiation pattern are presented to show the proposed antenna performance. Also, the ability of the pattern reconfigurability characteristics of the proposed antenna is well demonstrated.

\section{REFERENCES}

[1] J. Beutel, L. Harold, and L. Richard. Handbook of medical imaging. Vol. 1. Spie Press, 2000.

[2] S. A. Huettel, A. W. Song and G. McCarthy, Functional magnetic resonance imaging (Vol. 1). Sunderland, 2004.

[3] K. Doi, Computer-aided diagnosis in medical imaging: historical review, current status and future potential. Computerized medical imaging and graphics, 31(4-5), pp.198-211. 2007. 
[4] M. Guardiola, L. Jofre, F. Gedda, S. Capdevila, J. Romeu, and S. Blanch, 3D arrayed microwave tomographic system for medical imaging. In Wireless Information Technology and Systems (ICWITS), 2010 IEEE International Conference on, pp. 1-4, August 2010.

[5] A. Alqadami, S. Bialkowski, A. T. Mobashsher, and A. M. Abbosh. "Wearable Electromagnetic Head Imaging System Using Flexible Wideband Antenna Array Based on Polymer Technology for Brain Stroke Diagnosis." IEEE transactions on biomedical circuits and systems, 2018

[6] A. R. Mohammadi-Nejad, et al. Neonatal brain resting-state functional connectivity imaging modalities. Photoacoustics, 2018.

[7] M. Ojaroudi, S. Bila, and M. Salimi, "A Novel Approach of Brain Tumour Detection Using Miniaturized High-Fidelity UWB Slot Antenna Array", 13th European Conference on Antennas and Propagation (EUCAP),31 March-5 April, Krakow, Poland, 2019.

[8] A. T. Mobashsher, A. Mahmoud, and A. M. Abbosh. "Portable wideband microwave imaging system for intracranial hemorrhage detection using improved back-projection algorithm with model of effective head permittivity." Scientific reports 6, pp. 20459, 2016.

[9] E. Ricci, C. Ernestina, R. Tommaso, D. Marina, and D. Parth. "Performance evaluation of novel microwave imaging algorithms for stroke detection using an accurate 3D head model." Wireless Personal Communications 96, no. 3m pp. 3317-3331, 2017.

[10] Reimer, Tyson, Jordan Krenkevich, and Stephen Pistorius. "An OpenAccess Experimental Dataset for Breast Microwave Imaging." In 2020 14th European Conference on Antennas and Propagation (EuCAP), pp. 1-5. IEEE, 2020 .

[11] M. Ojaroudi, S. Bila, Ph. Leveque, and Ph. Carré. "Functional Microwave Imagining System based on Cognitive Scanning for Brain Activities Monitoring: A Feasibility Study." In 2019 13th European Conference on Antennas and Propagation (EuCAP), pp. 1-5. IEEE, 2019.

[12] M. Ojaroudi, and S. Bila, "Dynamic Short-Range Sensing Approach Using MIMO Radar for Brain Activities Monitoring", 14th European Conference on Antennas and Propagation (EUCAP), Copenhagen, Denmark, 2020. Copenhagen, Denmark15-20 March, 2020.

[13] M. Helbig, J. H. Koch, S. Ley, R. Herrmann, M. Kmec, K. Schilling, and J. Sachs, Development and test of a massive MIMO system for fast medical UWB imaging. In Electromagnetics in Advanced Applications (ICEAA), pp. 1331-1334, September 2017.

[14] S. A. Darvazehban, A. Zamani, and A. M. Abbosh, "Pattern Reconfigurable Metasurface Antenna for Electromagnetic Torso Imaging." IEEE Transactions on Antennas and Propagation, 2019.

[15] M. Ojaroudi, and S. Bila, "Multiple Moving Targets Tracking Based on Kernel Localization and Hierarchical Group Trackers for Functional Microwave Brain Imaging Applications", Accepted for publication in 14th European Conference on Antennas and Propagation (EUCAP), Copenhagen, Denmark, 15-20 March, 2020. Copenhagen, Denmark, 2020.

[16] CST Microwave Studio. ver. 2016, CST, Framingham, MA, USA, 2019. 